\title{
Analysis of Postoperative Outcomes in Patients with Intermittent Exotropia According to Preoperative Change of Angle of Deviation
}

\author{
Suji Hong, Sukgyu Ha, Youngwoo Seo, Sunghyun Kim \\ Department of Ophthalmology, Korea University College of Medicine, Seoul, Korea
}

Purpose: The correlation between the existence of the preoperative condition of $\geq 10$ prism diopters (PD) in patients with basic type of intermittent exotropia (IXT) and postoperative outcomes was analyzed.

Methods: The medical records of patients that underwent surgery for IXT were analyzed retrospectively. The analysis was conducted by dividing the patients into a group with change of <10 PD (group 1) and $\geq 10$ PD (group 2) before the time of the surgery. Patients who received at least 6 months of follow-up after surgery were included. The age, sex, angle of deviation and stereoacuity of the patients were studied. Surgical success was defined as exodeviation of $<10$ PD or esodeviation of <4 PD at the final visit after the surgery. The correlation between clinical factors and surgical success rate was analyzed by using correlative analysis.

Results: A total of 129 patients participated in the study. There were 108 (83.7\%) and 21 (16.3\%) patients in groups 1 and 2 , respectively. There were $89(82.4 \%)$ and $17(80.1 \%)$ patients with surgical successes in groups 1 and 2 , respectively $(p=0.18)$. Moreover, 13 (12.0\%) patients in group 1 and three (14.3\%) patients in group 2 required reoperation, showing no significant difference $(p=0.12)$. There was no statistically significant correlation between surgical success and preoperative change of angle of deviation $<10 \mathrm{PD}$ (odds ratio, 1.78; $p=0.17$ ).

Conclusions: Among the patients with basic type of IXT subjected to the analysis, $16.3 \%$ had a change of $\geq 10$ PD before surgery, and there was no significant correlation between surgical success and preoperative change of angle of deviation.

Key Words: Assessment, Exotropia, General surgery, Measures

Intermittent exotropia (IXT) without surgical treatment gradually progresses in frequency and angle of deviation and can eventually progress to constant exotropia. von Noorden and Campos [1] and Jampolsky [2] reported that

Received: November 2, 2020 Final revision: December 10, 2020

Accepted: December 17, 2020

Corresponding Author: Sukgyu Ha, MD, PhD. Department of Ophthalmology, Korea University Guro Hospital, 148 Gurodong-ro, Guro-gu, Seoul 08308, Korea. Tel: 82-2-2626-1260, Fax: 82-2-857-8580, E-mail: doctorha2@korea.ac.kr based on a longitudinal observation of patients with IXT between the ages of 5 and 10 years who had not received surgical treatment, $75 \%$ of the patients showed a progress in frequency and angle of deviation [1], and other study also reported a significant increase in short-distance angle of deviation based on a 2-year follow-up observation of patients who did not undergo surgical treatment [3].

Treatment options for IXT can be divided into non-surgical treatment, such as correction of refractive error, prism glasses, and occlusion therapy, and surgical treatment. Cases where patients have 15 to 20 prism of angle of 
deviation or greater, strabismus is seen in more than half of the waking hours, and there is marked decline in quality of life are indicated to surgical treatment [1]. While the recurrence rate after surgery in patients with IXT varied depending on the criteria for reoperation and study group [46], recurrence rates of $28.6 \%$ and $37.8 \%$ of have been reported based on longitudinal observations of 2 years or longer $[7,8]$.

Numerous studies investigated the effects of various factors affecting postoperative results of IXT patients by studying the effect of factors such as preoperative angle of deviation [9], myopic refractive error [10], difference in myopic and hyperopic angle of deviation, age at the time of surgery [11,12], and postoperative overcorrection [13,14] on surgical success. However, there was no study on the correlation between the preoperative change of IXT and postoperative outcomes.

This study aimed to analyze postoperative outcomes and investigate clinical impacts on surgical success according to the preoperative change of angle of deviation in patients with basic type of IXT.

\section{Materials and Methods}

This study was conducted after obtaining approval from the institutional review board of the Guro Hospital of Korea University (2020GR0171) and adhered to the Declaration of Helsinki. Written informed consent was waived due to the retrospective nature of the study. Medical records from patients who underwent unilateral or bilateral lateral rectus recession surgery between March 1, 2015 and December 31, 2019 for basic type of IXT diagnosed at Guro Hospital of Korea University.

Patients who underwent other ophthalmic surgeries, including different surgeries for strabismus, patients who underwent surgeries on oblique and superior/inferior rectus muscles, patients with lateral incomitancy, patients who had been diagnosed with neurological diseases, uncooperative patients and patients who had been observed for less than 6 months before or after surgery were excluded.

Sex, age, preoperative observation period, number of visits before surgery, refractive errors, preoperative near and distant angle of deviation, and near stereoacuity (Titmus; Stereo Optical, Chicago, IL, USA) of all patients were analyzed, and angles of deviation were examined at distant
$(6 \mathrm{~m})$ and near $(1 / 3 \mathrm{~m})$ by conducting alternate cover tests. The angle of deviation was measured using an alternating cover test (prism diopters, PD) and measured under the best-corrected visual acuity at each visit. The patients were observed at intervals of 2 to 12 months after surgery.

The surgeon (SGH) performed unilateral or bilateral lateral rectus recession based on the preoperative angle of deviation. Postoperatively, follow-up observations of patients were conducted after 1,3 , and 6 months, after which observations were conducted at 6-month intervals. Patients were divided into two groups based on the change of preoperative angle of deviation between at first and final visit prior to surgery: a group of patients with $\geq 10 \mathrm{PD}$ (group 1) and $<10$ PD (group 2) in distant angle of deviation.

Postoperative success was defined as an exodeviation of $<10$ PD and esodeviation of $<4$ PD based on the measurements at the time of the final visit. Surgical outcomes were compared between the two groups. Continuous variables were expressed as mean \pm standard deviation, and negative angle of deviation was presented as esodeviation.

The correlation between clinical variables, such as sex, age, number of visits before surgery, refractive error, preoperative distant and near angle of deviation, and stereoacuity, and the success of the surgery was analyzed to determine the influence of these clinical variables on the success of the surgery.

In the statistical analysis, IBM SPSS Statistics ver. 21.0 (IBM Corp., Armonk, NY, USA) was used. Mann-Whitney test and Fisher exact test were used to analyze the clinical variables of the two groups. Univariate logistic regression analysis was employed to analyze the correlation of clinical factors and success of surgery. A statistically significant difference was defined as a case where the $p$-value was $\leq 0.05$.

\section{Results}

Of the 129 patients enrolled in the study, 58 (45.0\%) were male, and $71(55.0 \%)$ were female, with a combined preoperative age of $9.4 \pm 7.1$ years (range, 3-20 years). The mean preoperative distant and near angles of deviation were 26.2 \pm 8.4 PD (range, 12-50 PD) and $26.8 \pm 8.1 \mathrm{PD}$ (range, 14$50 \mathrm{PD}$ ), respectively. The median stereopsis was $1.90 \mathrm{log}$ arcsec (range, 1.60-2.30 log arcsec), and the patients visited $3.1 \pm 1.8$ times (range, $3-7$ times) at $7.7 \pm 4.5$ months (range, 
Table 1. Comparison of clinical characteristics according to change of angle of deviation before surgery

\begin{tabular}{|c|c|c|c|}
\hline & Group $1(n=108)$ & Group $2(n=21)$ & $p$-value \\
\hline Age (yr) & $9.8 \pm 7.2(4-20)$ & $8.6 \pm 6.3(3-18)$ & $0.39^{*}$ \\
\hline Male & $48(44.4)$ & $10(47.6)$ & $0.34^{\dagger}$ \\
\hline Refractive error (D) & $-2.4 \pm 3.9(-4.7-2.1)$ & $-2.5 \pm 5.1(-3.6-3.8)$ & $0.37^{*}$ \\
\hline Preoperative follow-up period (mon) & $7.8 \pm 1.2(6-12)$ & $6.9 \pm 1.8(6-10)$ & $0.13^{*}$ \\
\hline \multicolumn{4}{|l|}{ Preoperative change of angle of deviation (PD) } \\
\hline Distant & $5.2 \pm 1.3(0-8)$ & $12.5 \pm 1.7(10-14)$ & $0.25^{*}$ \\
\hline Near & $5.1 \pm 1.9(0-8)$ & $11.9 \pm 2.0(10-18)$ & $0.22^{*}$ \\
\hline \multicolumn{4}{|l|}{ Preoperative angle of deviation (PD) } \\
\hline Distant & $27.1 \pm 7.5(12-50)$ & $29.3 \pm 6.7(20-40)$ & $0.43^{*}$ \\
\hline Near & $27.4 \pm 7.4(14-50)$ & $28.6 \pm 9.6(14-40)$ & $0.55^{*}$ \\
\hline \multicolumn{4}{|l|}{ Postoperative angle of deviation at POD 1 (PD) } \\
\hline Distant & $-12.1 \pm 8.4(-20-2)$ & $-11.5 \pm 8.8(-15-8)$ & $0.17^{*}$ \\
\hline Near & $-13.3 \pm 8.8(-20-4)$ & $-14.7 \pm 7.8(-18-6)$ & $0.11^{*}$ \\
\hline \multicolumn{4}{|l|}{ Postoperative angle of deviation at the final visit (PD) } \\
\hline Distant & $7.1 \pm 8.9(-10-25)$ & $6.4 \pm 9.7(-8-20)$ & $0.13^{*}$ \\
\hline Near & $7.9 \pm 9.1(-14-25)$ & $7.1 \pm 8.5(-12-18)$ & $0.11^{*}$ \\
\hline Median stereoacuity at the final visit (log arcsec) & $1.90(1.60-2.30)$ & $1.90(1.60-2.30)$ & $0.99^{*}$ \\
\hline Postoperative follow-up periods (mon) & $21.1 \pm 6.5(12-60)$ & $23.1 \pm 8.7(12-54)$ & $0.44^{*}$ \\
\hline Reoperation & $13(12.0)$ & $3(14.3)$ & $0.12^{\dagger}$ \\
\hline Success & $89(82.4)$ & $17(80.1)$ & $0.18^{\dagger}$ \\
\hline
\end{tabular}

Refractive error is calculated as spherical equivalent, minus value means myopia or esodeviation; Values are presented as mean \pm standard deviation (range), number (\%), or median (interquartile range). $\mathrm{D}=$ diopters; $\mathrm{PD}=$ prism diopters; POD 1 = postoperative day 1 .

"Mann-Whitney test; ${ }^{\dagger}$ Fisher exact test.

6-12 months) before surgery.

There were 108 patients (83.7\%) in group 1 and 21 $(16.3 \%)$ in group 2 . The age was not significantly different between the two groups $(p=0.39)$ as the age was $9.8 \pm 7.2$ years (range, $4-20$ years) in group 1 and $8.6 \pm 6.3$ years (range, $3-18$ years) in group 2 . The mean preoperative distant and near angles of deviation were 27.1 $\pm 7.5 \mathrm{PD}$ (range, 12-50 PD) and 27.4 $\pm 7.4 \mathrm{PD}$ (range, 14-50 PD), respectively, in group 1 and $29.3 \pm 6.7 \mathrm{PD}$ (range, 20-40 PD) and $28.6 \pm 9.6$ PD (range, 14-40 PD), respectively, in group 2, exhibiting no significant difference between the groups ( $p$ $=0.43$ and $p=0.55$, respectively). The angles of deviation on postoperative day 1 was $-12.1 \pm 8.4 \mathrm{PD}$ (range, $-20-2$ PD) in Group 1 and $-11.5 \pm 8.8$ PD (range, $-15-8$ PD) in group 2, showing no significant different between the groups $(p=0.43)$. The postoperative follow-up periods were $21.1 \pm 6.5$ months (range, $12-60$ months) in group 1 and $23.1 \pm 8.7$ months (range, $12-54$ months) in group $2(p$ $=0.44)$. The postoperative distant angles of deviation at the final visit were $7.1 \pm 8.9 \mathrm{PD}$ (range, $-10-25 \mathrm{PD}$ ) in group 1 and $6.4 \pm 9.7 \mathrm{PD}$ (range, $-8-20 \mathrm{PD}$ ) in group $2(p=0.13$ ). During the follow-up period, there was no statistically significant difference in the reoperation rate between the groups, as thirteen (12.0\%) patients in group 1 and three $(14.3 \%)$ patients in group 2 underwent reoperation ( $p=$ 0.12 ). In group 1 , twelve patients underwent reoperation for recurrent exotropia $>15 \mathrm{PD}$, only one patient for consecutive esotropia $>10$ PD during follow-up period. In group 2, all of three patients underwent reoperation for recurrent exotropia $>15$ PD during follow-up period. There were 89 $(82.4 \%)$ and $17(80.1 \%)$ patients with surgical successes in groups 1 and 2, respectively ( $p=0.18$ ) (Table 1). Details of preoperative and postoperative clinical data are showed in Table 1 and Fig. 1. 


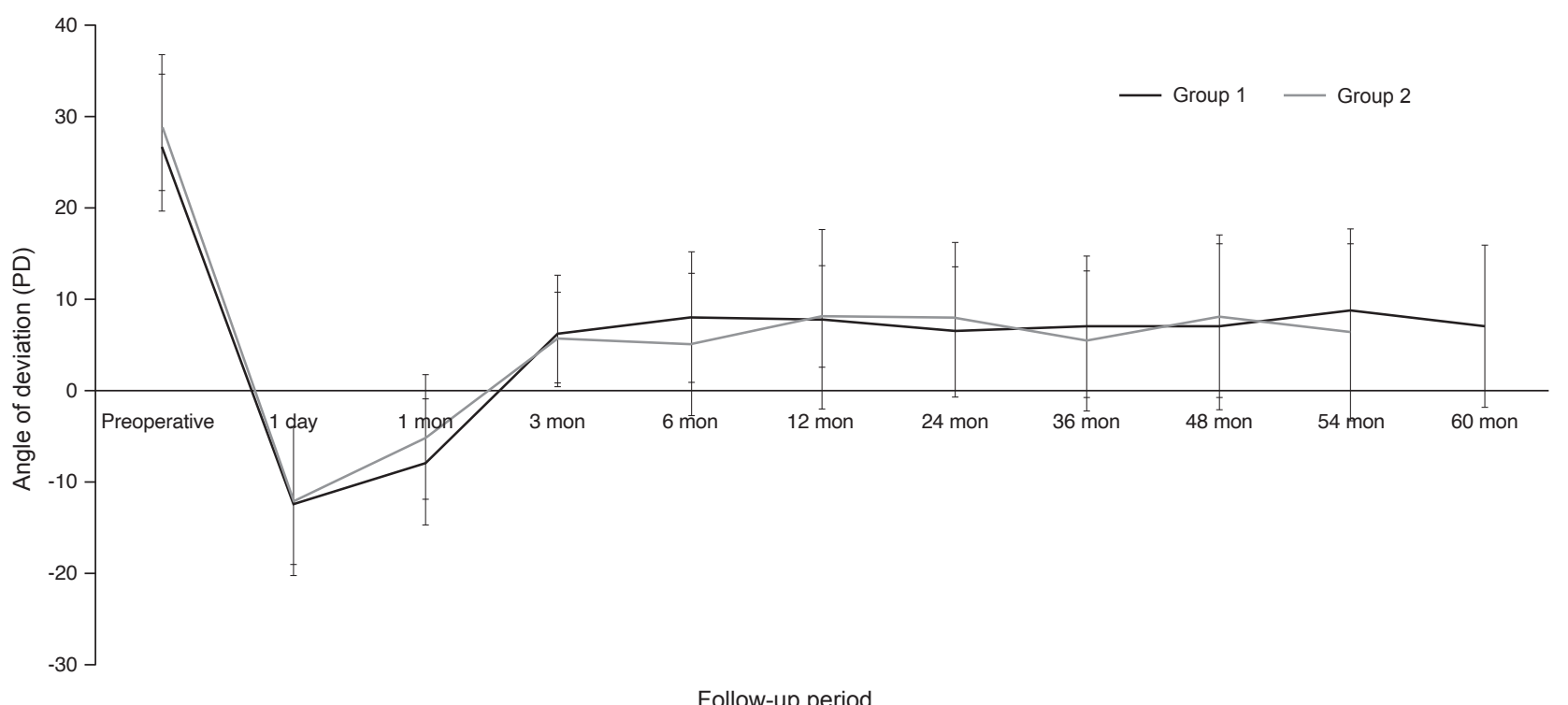

Fig. 1. The distant angle of deviation of two groups during follow-up period. $P D=$ prism diopters.

Univariate logistic regression analysis revealed that patient's age, sex, refractive error, preoperative angle of deviation, and change of angle of deviation ( $<10$ PD) before surgery upon the success of the surgery were analyzed. Age at the time of surgery (odds ratio [OR], $0.11 ; p=0.21$ ), sex (OR, $0.32 ; p=0.54)$ and refractive error (OR, $0.84 ; p=$ 0.32 ) did not have a significant correlation with the success of surgery. Preoperative angle of deviation had no significant association with the success of surgery (OR, $0.14 ; p=$ 0.43 ). The angle of esodeviation on postoperative day 1 had significant effect on the surgical success (OR, 2.14; $p=$ $0.03)$. Whether the change of deviation ( $<10$ PD) before surgery was did not have a statistically significant effect on the success of the surgery (OR, $1.78 ; p=0.12)$.

\section{Discussion}

There was no statistically significant difference in the success and reoperation rates after surgery between the patient group with IXT $\geq 10$ PD increase in angle of deviation and patient group with an increase of $<10$ PD prior to surgery. There was no statistically significant correlation between the success rate of surgery and change in angle of deviation observed during the preoperative period.

The clinical effects of various factors on the outcome of the surgery for IXT, including studies on preoperative angle of deviation [9], myopia [10], preoperative angle of deviation, age at the time of surgery [11,12], and postoperative overcorrection [12-14]. Particularly, the study by Gezer et al. [15] showed that the success rate of surgery increases when the preoperative angle of deviation is lower, but there have been no studies the effect of change of angle of deviation during a certain preoperative period on surgical outcome in patients with IXT. The IXT has been reported to exacerbate as the angle of deviation and frequency of deviation increases over time [1,2], but the appropriate timing of surgery has been controversial.

Incidence of consecutive esotropia and recurrence following surgery for IXT could be increased when patients are younger at the time of the surgery $[1,12]$ and prevalence of amblyopia and deterioration stereopsis after surgery were lower in the patient group with age $>4$ years. However, another study also showed that the success rate of surgery was higher in patients who were aged $\leq 4$ years [8], and that the success rate improved when the surgery was performed at an earlier point of time [16]. Other studies reported that the success rate of surgery was significantly lower when there was preoperative decline in stereopsis $[17,18]$, but there was no significant correlation found in this study.

Previous studies reported that postoperative overcorrec- 
tion is a factor that lowers reoperation rate in patients with IXT. Regarding postoperative overcorrection that shows a high success rate, studies have investigated various degrees of overcorrection, such as $>10$ PD and $>10-20$ PD $[13,19,20]$. In this study, among all clinical variables used in this study, overcorrection on the day after the surgery was found to be the only factor that has significant correlation with the success of the surgery.

In surgery for IXT, there are several factors to be considered, but when the angle of deviation progresses rapidly during the preoperative observation period, it can be difficult to predict the surgical outcome. In addition, when determining the amount of surgery based on the time of surgery and angle of deviation, if the angle of deviation progresses rapidly during the observation period, determining the time of surgery and required amount of surgery can become challenging. This study compared the surgical outcomes based on varying rates of preoperative change of angle of deviation in patients undergoing surgery for basic type of IXT. This study has demonstrated that the preoperative change of angle of deviation does not have a significant effect on the surgical outcome by comparing the surgical outcomes of patients who had change of $\geq 10$ PD to those of patients who had change of $<10$ PD before the surgery.

There are several limitations in this study. The first limitation of this study was that it only targeted patients with basic type of IXT. This study did not include patients with divergence excess or convergence insufficiency IXT. Future studies that include other types of IXT will be necessary. Second, this study was analyzed by e retrospective design. A prospective study on this will be needed in the future.

Of patients with IXT, $16.3 \%$ had change of $\geq 10$ PD before surgery. The preoperative change of angle of deviation did not affect the postoperative recovery and reoperation rate. The authors believe that the results of this study will aid in the prediction of prognosis of IXT surgery and that the preoperative change of angle of deviation in IXT has a minimal impact on the prognosis after surgery. This study can aid clinician illustrating the prognosis following surgery to patients and their carers who are experience great anxiety from the rapid change of the angle of deviation.

\section{Conflict of Interest}

No potential conflict of interest relevant to this article was reported.

\section{References}

1. von Noorden GK, Campos EC. Exodeviation. In: von Noorden GK, Campos EC. Binocular vision and ocular motility: therapy and management of strabismus. 6th ed. St Louis: CV Mosby; 2002. p. 358-9.

2. Jampolsky A. Characteristics of suppression in strabismus. AMA Arch Ophthalmol 1955;54:683-96.

3. Jung MS, Choi MY. The natural course in children with intermittent exotropia. J Korean Ophthalmol Soc 2003;44: $1572-7$.

4. Han DH, Paik HJ. The minimal postoperative follow-up period to determine secondary surgery in patients with intermittent exotropia. J Korean Ophthalmol Soc 2014;55:7118.

5. Kim MJ, Kim SH. Factors associated with improved surgical outcomes in recurrent exotropia. J Korean Ophthalmol Soc 2017;58:692-7.

6. Ekdawi NS, Nusz KJ, Diehl NN, Mohney BG. Postoperative outcomes in children with intermittent exotropia from a population-based cohort. J AAPOS 2009;13:4-7.

7. Richard JM, Parks MM. Intermittent exotropia: surgical results in different age groups. Ophthalmology 1983;90:1172-7.

8. Cho YA, Lee JK. Early surgery before 4 years of age in intermittent exotropia. J Korean Ophthalmol Soc 2004;45:6205.

9. Scott AB, Mash AJ, Jampolsky A. Quantitative guidelines for exotropia surgery. Invest Ophthalmol 1975;14:428-36.

10. Zou D, Casafina C, Whiteman A, Jain S. Predictors of surgical success in patients with intermittent exotropia. $J A A$ POS 2017;21:15-8.

11. Keenan JM, Willshaw HE. The outcome of strabismus surgery in childhood exotropia. Eye (Lond) 1994;8:632-7.

12. Lee JY, Choi DG. The clinical analysis of recurrence after surgical correction of intermittent exotropia. $J$ Korean Ophthalmol Soc 2002;43:2220-6.

13. Paik HJ, Sohn HJ. Early surgery for intermittent exotropia in children less than 4 years of age. $J$ Korean Ophthalmol Soc 2006; 47:477-83.

14. Kim SJ. Comparison of surgical results between bilateral 
recession and unilateral recession-resection in intermittent exotropia. J Korean Ophthalmol Soc 1992;33:733-8.

15. Gezer A, Sezen F, Nasri N, Gozum N. Factors influencing the outcome of strabismus surgery in patients with exotropia. $J$ AAPOS 2004;8:56-60.

16. Edelman PM, Brown MH, Murphree AL, Wright KW. Consecutive esodeviation: then what? Am Orthoptic $J$ 1988;38:111-6.

17. Beneish R, Flanders M. The role of stereopsis and early postoperative alignment in long-term surgical results of in- termittent exotropia. Can J Ophthalmol 1994;29:119-24.

18. Gill MK, Drummond GT. Indications and outcomes of strabismus repair in visually mature patients. Can J Ophthalmol 1997;32:436-40.

19. Raab EL, Parks MM. Recession of the lateral recti: early and late postoperative alignments. Arch Ophthalmol 1969;82:203-8.

20. Knapp P, Helveston EM, Jampolsky A, et al. Transections of the New Orleans Academy of Ophthalmology: symposium on strabismus. St Louis: CV Mosby; 1971. p. 233-41. 